Kürzere Beiträge

\title{
Kolloquium zur Schweizer Pestgeschichte
}

Am 3. Juni 1978 fand in Basel das zweite Kolloquium über «Pest und Pestbekämpfung in der Schweiz » statt. Es wurde veranstaltet vom Historischen Seminar der Universität Basel (Prof. Dr. phil. M. Mattmüller, Dr. phil. H.R.Burri) und vom Medizinhistorischen Institut der Universität Zürich (Prof. Dr. med. H.M. Koelbing, Dr. phil. H.P. Ruesch).

Seit mehreren Jahren stehen das Basler Seminar und das Zürcher Institut in einem interdisziplinären Gespräch, das an die unerklärte Tatsache anknüpft, daß im 18. Jahrhundert die Einwohnerzahl der Schweiz stark ansteigt. Als Ursache vermuteten frühere Forscher das Ausbleiben der Pestzüge. Die Basler Historiker stellen diese Hypothese auf Grund der bisher zur Bevölkerungsentwicklung im 16., 17. und 18. Jahrhundert gewonnenen Erkenntnisse in Frage und ziehen andere Ursachen, beispielsweise die bessere Ernährung, in Betracht. Gerade deshalb ist es ihr Anliegen, die Pest und ihre demographisch faßbaren Folgen genauer zu überprüfen und die Auswirkungen auf das soziale, wirtschaftliche und politische Leben aufzuzeigen. Die Medizingeschichte sieht in den behördlichen und ärztlichen Maßnahmen, die allenfalls das Aufhören der Pest gefördert oder gar bedingt haben könnten, ein lohnendes Thema.

Zur Überprüfung der Ausgangslage und zur Rekapitulation der Pestbiologie wurde am 14.Juni 1975 ein erstes pesthistorisches Kolloquium in Zürich abgehalten. Ein im Anschluß daran aufgestelltes Forschungsprogramm, das die Unterstützung des Schweizerischen Nationalfonds genießt, wird von einer Reihe von Doktoranden und Mitarbeitern des Medizinhistorischen Instituts Zürich getragen. Auch die 16 am zweiten Kolloquium vom Juni 1978 vorgelegten Arbeiten beweisen, daß die 1975 an die Forschung gestellten Fragen die Aufmerksamkeit von Historikern und Medizinhistorikern im In- und Ausland auf die Pest zu lenken vermochten. Neun Lokalstudien befassen sich mit den Pestzügen des 16. und 17. Jahrhunderts in der Ostschweiz, im Baselbiet, in Genf, Solothurn und Mainz. Besonders erwähnt sei das Referat von P. Dubuis, der ein Register aus dem Jahre 1349 analysiert, in dem 389 Todesfälle aus der Stadt und Pfarrei St-Maurice im Wallis verzeichnet werden. Die bisher unbekannte Quelle ist um so bedeutsamer, als Namenslisten der Opfer des «Schwarzen Todes» sehr selten sind. Sieben weitere Beiträge gehen auf die medizinischen Auffassungen und die behördlichen Maßnahmen ein. Beachtung verdienen hier die Ausführungen von Frau A. Stettler über die für die nachträgliche Diagnosestellung wichtige Entwicklung des Begriffs «Pest» von einer Seuche mit hoher Sterblichkeit zur ätiologisch und pathogenetisch definierten Infektionskrankheit.

334 
Einige Schwierigkeiten des interdisziplinären Gesprächs traten in der Diskussion zutage, die die Kolloquiumsteilnehmer aus der Schweiz, der Bundesrepublik Deutschland, aus Frankreich und den USA unter der Leitung der Professoren Koelbing und Mattmüller führten. So bereitet es nicht geringe Mühe, die von den Epidemiologen vertretenen Auffassungen über das Wesen und die Übertragungsmechanismen der Pest mit den Resultaten der Historiker und den zeitgenössischen Berichten in Einklang zu bringen. Zur Theorie, daß die Pest primär eine Krankheit der Nager - der Ratten - ist, die durch Flöhe auf den Menschen übertragen wird, scheint zwar sehr gut die Feststellung von E.Menolfi zu passen, daß in Sulgen (Thurgau) in den Jahren 1629 und 1630 besonders die Kornbauern und Müller an der Pest starben (Getreide - Ratten). Wie läßt sich aber erklären, daß die Seuchenzüge (die Ratten?) an militärisch gesperrten Grenzen Halt machen? Ist nicht die Verbreitung durch Bettler und Vagabunden, durch Kleider, Hausrat und Warentransporte in unzähligen Fällen belegt, keineswegs aber Rattensterben? Muß nicht die Folgerung erlaubt sein, daß die Übertragung von Mensch zu Mensch eine Hauptrolle spielt? Während der Bakteriologe Prof. Dr. med. Hans Reber fordert, daß Historisches von experimentell gesicherten Tatsachen aus interpretiert werde, äußern andere Zweifel, ob die heutigen biologischen Gegebenheiten auch vor 500 Jahren galten. Ein Konsens konnte in der Diskussion zumindest in diesen Fragen nicht erreicht werden, Grund genug, den begonnenen Dialog fortzusetzen. Gerade die behördlichen Maßnahmen, die Auswirkungen der Reise- und Handelssperren, die weiteren Folgen für die Organisation der öffentlichen Gesundheitspflege wie auch die ärztlichen Pesttraktate verdienen genauer betrachtet zu werden. Die derzeit laufenden Studien des Zürcher Forschungsprogramms befassen sich denn auch vorwiegend mit diesem Themenkreis. Die Ergebnisse werden in zwei bis drei Jahren Stoff zu einem dritten Kolloquium liefern. Auch die bisher strittigen Fragen sollten in genauer Kenntnis des jeweils anderen Standpunktes und im Bewußtsein der Grenzen der einzelnen Methoden erneut besprochen werden. Hier zwischen Historikern und Medizinern zu vermitteln, hat die Medizingeschichte als Aufgabe erkannt. Daß eine die Forschungsresultate zusammenfassende Darstellung des Phänomens «Pest in der Schweiz» das Gespräch zwischen den Fakultäten kröne, ist sehr zu hoffen.

Urs Boschung

\section{Anmerkung}

$\mathrm{Ob}$ und in welcher Form die am Basler pesthistorischen Kolloquium vorgelegten Arbeiten gedruckt werden, ist noch offen. Vervielfältigungen, wie sie jeder Tagungsteilnehmer ausgehändigt erhielt, sind in wenigen Exemplaren noch erhältlich beim Historischen Seminar der Universität Basel, Hirschgäßlein 21, 4051 Basel, und beim Medizinhistorischen Institut der Universität Zürich, Rämistraße 71, 8006 Zürich. 


\section{Halleriana}

\section{Eine Reminiszenz an die Bibliothek A.v.Hallers}

In den ersten Herbsttagen 1778 bewegte sich ein ungewöhnlicher Zug von 80 schwerbepackten Maultieren das obere Reußtal hinauf über die Gotthardstraße. Die Saumtiere trugen auf ihrem Rücken die wissenschaftliche Ausbeute eines langen und rastlosen Gelehrtenlebens, die Bibliothek und die eigenen gedruckten Werke samt zahlreichen Handschriften des rund 8 Monate vorher verstorbenen Albrecht von Haller von seiner Heimatstadt nach Italien.

Kaiser Joseph II. hatte auf der Rückreise aus Frankreich am 17. Juli 1777 den kranken Gelehrten in Bern besucht. Bald nach dessen Tod bemühte man sich von Wien aus, seinen wissenschaftlichen Nachlaß, der unter den privaten Büchersammlungen Europas als einer der vorzüglichsten galt, für die Bibliothek der Brera (Braidense) im damaligen österreichischen Mailand zu erwerben, wo unter Maria Theresia der Jesuitenorden den Palazzo dieses Namens hatte räumen müssen.

Der am Hofe zu Wien allmächtige Minister und Reichsfürst von Kaunitz-Rittberg erbat sich deshalb im April 1778 von Hallers ältestem Sohn Gottlieb Emanuel den Katalog der Bibliothek. Er äußerte den Wunsch, sie zu erwerben, weil Werke aus dem Gebiet der Medizin, Chirurgie, Anatomie, Botanik und Naturgeschichte in der Brera fehlten. Gottlieb Emanuel erklärte im Brief vom 10. Mai im Namen der Familie die Bereitwilligkeit zu einem Verkauf des insgesamt 13512 Nummern umfassenden Bestandes.

\begin{tabular}{lr} 
Libri anatomici & 1874 \\
Libri chirurgici & 953 \\
Libri pratici & 3094 \\
Libri botanici & 1596 \\
Libri di storia naturale & 2470 \\
Giornali o Memorie & \\
\multicolumn{1}{l}{ delle accademie } & 1677
\end{tabular}

\begin{tabular}{lr} 
Varie & 1253 \\
Volumi di disputi interno & \\
$\quad$ a tali scienze & 350 \\
Delle Erbe vive & 100 \\
Autografi dell'Haller & \\
$\quad$ in parte inediti & 145 \\
\hline & 13512
\end{tabular}

Über den Kaufpreis schrieb er, sein Vater habe die gut erhaltene Sammlung auf 60000 französische Lire geschätzt. Man sei bereit, sie billiger abzugeben, doch angesichts von Interessenten aus Rußland und England nicht unter 2000 neuen Louisd'or, zahlbar bei der Übernahme. Kaunitz fand den Preis angemessen, nicht nur wegen der Reichhaltigkeit der während rund eines halben Jahrhunderts gesammelten Werke, sondern auch wegen der wertvollen Handschriften, des berühmten Herbariums und der noch nicht veröffentlichten Manuskripte. 
Da die Berner Behörden gegen einen Verkauf ins Ausland nicht opponierten, erklärten die Erben Hallers am 28. Brachmonat 1778, das Angebot von 2000 Louisd'or für die Bibliothek ihres Gatten und Vaters anzunehmen. Darauf trafen am 11.Juli 1778 zwei Bevollmächtigte der österreichischen und lombardischen Regierung nach einer elftägigen und mühseligen Reise in Bern zur eingehenden Überprüfung der Bestände auf Grund des erhaltenen Kataloges ein. Zu Anfang August war die Inspektion und Verpackung beendet. Die Verkaufsquittung trägt das Datum des 28. Juli. Die 156 Kisten - jede auf 150 Pfund abgewogen, damit je 2 Kisten die Ladung für ein Maultier ausmachten - nahmen nun ihren Weg durch das bernische Emmental nach Luzern und über den Gotthard nach Mailand, wo sie Anfang Oktober in bestem Zustand eintrafen.

Paul Röthlisberger

\section{Der Gedenkstein für Hallers jüngste Tochter}

Man fährt von Bern mit der Schwarzenburgbahn nach Niederscherli, überschreitet die Brücke über den tief eingeschnittenen Scherlibach und steigt über Bifit aufwärts nach Farneren. Dieser Name weist auf die Farnkräuter jener früher wilden Landschaft hin. Die weite Mulde wurde im Juni 1799 von Hallers Tochter Charlotte, der Witwe des Bankiers Ludwig Zeerleder, erworben, und der Gatte ihrer Stieftochter aus erster Ehe ihres Mannes, Architekt Ahasver Carl von Sinner, baute für sie den oberen der beiden Höfe. Dahinter liegt ein Wäldchen, das durch ein Weglein von rechts her zugänglich ist. Einige Meter unterhalb jenes Pfades entdeckt man im Waldesinnern zwei mannshohe Gedenksteine, deren Vorderseite nach dem Farnerengut gerichtet ist. Der Stein rechts trägt oben im Halbrund das Bild eines Insekts mit dem gewölbten Spruch: «Non tota perit» (Sie vergeht nicht ganz), darunter die gut erhaltene Inschrift: «Dem heiligen Andenken / an die beste der Mütter, / an die edle, vortreffliche / Frau Sophie Charlotte / Zeerleder, / gebohrne von Haller, / der Stifterin dieser aus / einer Wildniss in einen / blühenden Garten / verwandelten lieblichen / Gegend, weihet dankbar / ihr Sohn Albert / mit gerührtem Herzen / diesen Platz, / und mit stillen Thraenen, / mit bewegtem Gemüth, / sezt er der Seeligen / diesen Stein. / Des Sohnes Seele trauert / für die, ach, zu früh / verlohrne liebe Mutter / und bewundert / den schönen Seegen, / den der Himmel auf diesen / der vormahligen Besitzerin / so werthen Gefilden / walten läßt. / MDCCCXIV.» Charlotte war 1805 gestorben; der Stein wurde 1814 von ihrem Erben gesetzt. Dieser selbst hat Berns Untergang 1798 als 22jähriger Schützenleutnant in Laupen und beim siegreichen Kampf in Neuenegg miterlebt und über die Ereignisse jener Tage einen der wertvollsten Quellenberichte verfaßt, den Richard Feller im 4.Band seiner Geschichte Berns oft zitiert. Dieser Enkel Hallers hieß Albrecht, wurde aber Albert genannt. Er 
erhielt den Gedenkstein zur Seite seiner Mutter mit der Inschrift: «Albert Zeerleder / geb. den 28 December 1776, / gest. den 23 Februar 1825, / folgte der / vortrefflichen Mutter / in dem Besize dieses Gutes / gleich-wie / in Übung stiller Tugend. / Dieses Andenken / setzte sein dankbares Geschlecht, / das er zu seinem Erbe berufen.»

Eine wertvolle Biographie Charlotte Zeerleders von René Moeri erschien in der «Berner Zeitschrift für Geschichte und Heimatkunde», Jg. 36 (1974), Heft 1. Ihm und Herrn Dr. Wolfgang Gresky danke ich für die Wegweisung zu der wenig bekannten geschilderten Stätte.

Heinz Balmer

3. Albrecht von Haller der Jüngere (1758-1823): zu seiner Würdigung und zur Frage seines Porträts

Albrecht von Haller filius erbte von seinem Vater nicht nur die Ähnlichkeit der Gesichtszüge und die Größe der Gestalt, sondern auch die Urteilskraft des Staatsmannes und die Liebe zur Botanik, ferner ein gutes Gedächtnis und eine Gabe zu dichten. Die ausführlichste Biographie (von John Briquet in dessen «Biographies de botanistes suisses», Genève 1906) würdigt den Naturforscher und behauptet, als solcher habe er tiefere Spuren hinterlassen denn als Staatsmann. In Richard Fellers «Geschichte Berns» tritt er dagegen als «vielseitig verwendetes Ratsmitglied» auf, das heiklen Aufgaben in schwerer Zeit mit Festigkeit und Würde gewachsen war.

Haller filius war der umfassendste Botaniker Berns in seiner Zeit; er gehörte zu den Trägern der ersten Berner Naturforschenden Gesellschaft, schuf und leitete den botanischen Garten, lehrte sein Fach als Professor am medizinischen Institut und hinterließ sein reiches, hervorragendes Herbar schweizerischer Pflanzen der Bibliothek Genf, was dort den Anstoß zur Errichtung des ersten botanischen Museums gab und noch um 1900 mehrere Publikationen über darin enthaltene Pflanzengruppen auslöste (Rosen, Habichtskräuter, Enziane und Gräser). Selber hat Haller filius vor allem zu Jean Gaudins «Flora helvetica» und zur 16. Auflage von Linnés «Systema vegetabilium», die Johann Jakob Römer und August Schultes besorgten, Beiträge geliefert. John Briquet zählt die Gegenden auf, in denen Haller filius zwischen 1775 und 1822 botanisierte; auch nennt er über 50 Botaniker, mit denen er in Briefwechsel stand. Gaudin findet in seiner «Flora helvetica» (Band 1, Zürich 1828, S. XXVII und 262) Worte tiefster Dankbarkeit und Verehrung für den vir optime meritus, der ihm während zwanzig Jahren unschätzbare Dienste geleistet habe.

Haller filius wurde 1779 Kriegsratsschreiber, 1795 Mitglied des Großen Rates mit der Funktion eines Geleitsherrn. So hatte er 1797 den durchreisenden Bona- 
parte von Bern über Jegenstorf und Fraubrunnen nach Solothurn zu begleiten. Haller durchschaute ihn und tat in der Folge alles, um Berns Untergang zu verhindern. Als die Franzosen im Dezember 1797 den Südjura besetzten, ließ er sich zum französischen Geschäftsträger Theobald Bacher nach Basel abordnen und erhob Einspruch. Als er anfangs 1798 die Sicherheit des Staates durch das Zaudern der bernischen Regierung in Gefahr sah, stellte er den Antrag, die Staatsgewalt während der Dauer des Notstandes einem Siebenmännerausschuß zu übertragen. Dies wurde ernsthaft erwogen, aber verworfen. Da die luzernischen Truppen gegen die anrückenden Franzosen nicht kämpfen wollten, eilte Haller nach Luzern, um an die alte Bundespflicht zu mahnen.

Es war nicht seine Schuld, daß Bern unterging. Später anerkannte man seine Voraussicht. Er gelangte 1814 in den Kleinen Rat und wirkte 1816-1821 als Oberamtmann zu Interlaken. 1822 präsidierte er die Jahresversammlung der Schweizerischen Naturforschenden Gesellschaft in Bern und hielt eine überzeugende Rede über den Sinn und Wert naturwissenschaftlicher Sammlungen. Im «Burger Todtenrodel II » im Staatsarchiv Bern ist sein Tod am 1. März 1823 eingetragen.

Nur die Sorge um das Vaterland hatte den sonst im persönlichen Umgang verschlossenen Mann seine Hemmungen überwinden lassen. Lange ging er seinen Weg allein. Er hat erst mit 50 Jahren eine Witwe geheiratet und blieb kinderlos. Seine Frau, Elisabeth Fischer von Reichenbach, eine Ururenkelin des Stifters der bernischen Post, hatte aus erster Ehe mit Samuel Gruner (1760-1788) zwei erwachsene Kinder, Sohn und Tochter; ein zweiter Sohn war 1803 gestorben. Der Sohn Emanuel Gruner (1783-1863) führte die Papierfabrik seines Vaters in Worblaufen weiter und war seit 1804 mit Julie von Jenner, einer Urenkelin des großen Haller, verheiratet, so daß Haller filius zugleich Stiefvater Gruners und Großonkel von dessen Frau war. Das Ehepaar Gruner-von Jenner hatte zwölf am Leben gebliebene Kinder. Durch sie wurde die Frau von Haller filius Ahnmutter eines verzweigten Gelehrtengeschlechtes. Außerdem wurde sie durch ihre Tochter Großmutter des in Paris bei Thenard und Dumas ausgebildeten Chemikers Ludwig Rudolf von Fellenberg, der Mineralwässer, Mineralien, Erze, antike Bronzen und Gläser analysierte und einige Zeit Professor in Lausanne war; seine Brüder leiteten die Evangelische und die Ökonomische Gesellschaft, und sein Sohn Edmund wurde als Alpengeologe und Archäologe bekannt.

Elisabeth Fischers zweiter Lebensgefährte aber verschwand im Schatten seines großen Vaters. Die besonderen Umstände seines Lebens als Staatsmann in einer Untergangszeit, als selbstloser Helfer anderer Forscher und als übersorgfältige, zurückhaltende Persönlichkeit gaben ihn fast ganz der Vergessenheit preis.

Ein einziges Porträt überliefert uns seine vornehmen Züge. Es handelt sich um 
eine schöne Lithographie seines Zeitgenossen, des Obwaldner Kirchenmalers und Porträtisten Joseph Anton Heimann (1758-1837), die in der lithographischen Anstalt, die der Stuttgarter Georg Adolf Grimminger 1833 in Zürich eröffnete, vervielfältigt wurde. Die Wiedergabe bei Briquet stützt sich auf die Vorlage in der Zentralbibliothek Luzern. Die Burgerbibliothek Bern, die übrigens viele Briefe von Haller filius besitzt, bewahrt ebenfalls ein Exemplar der Lithographie auf. Darunter hat Haller filius einen seiner Verse geschrieben:

«Ein wohlgesetzt Gemüth kann Galle süße machen, da ein verwöhnter Sinn auf alles Wermuth streut»; in Klammern hat er seinen Namen «(Albr. Haller)» beigefügt.

Aber selbst dieses Porträt ist Haller entzogen worden. Friedrich Haag hat 1914 in seinem Werk über «Die Sturm- und Drang-Periode der Bernischen Hochschule 1834-1854» (bei Seite 50) das Haller-Bildnis irrtümlich als Kasthofer-Bildnis wiedergegeben. Forstmeister Karl Albrecht Kasthofer (1777-1853) war ebenso wie Haller Bernburger, Politiker, Botaniker und mit dem Berner Oberland verbunden. Äußerlich aber waren sie sehr verschieden. Auch von Kasthofer gibt es ein einziges Bildnis, ein ovales Ölgemälde im Besitz der Philosophischen Fakultät der Universität Bern. Wiedergegeben ist es am Anfang der Kasthofer-Biographie Jakob Sterchis in der «Sammlung Bernischer Biographien», Band 5, S.528. Dort steht (S.550) ausdrücklich, es sei Kasthofers einziges Bild; Gustav Tobler (der kundige Historiker und Herausgeber von Kasthofers Selbstbiographie) habe es vermittelt. Der zierliche Kasthofer nannte sich schon mit 49 Jahren alt und schwach (siehe bei Sterchi S.546), während Hallers «mächtiger Körperbau » von Bernhard Studer, der ihn gut kannte, in seiner «Geschichte der Physischen Geographie der Schweiz bis 1815» (S.453) bezeugt wird. Glücklicherweise ist die von Walter Boßhard herausgegebene Kasthofer-Anthologie: «Wer Bäume pflanzt ... der wird den Himmel gewinnen» (Eidg. Anstalt für das forstliche Versuchswesen, Bericht Nr.174, Birmensdorf 1977) mit dem richtigen Kasthofer-Bildnis geschmückt. Dagegen hat man im «Jahrbuch vom Thuner- und Brienzersee 1973 » eine Arbeit über Kasthofer mit einem Ausschnitt aus der Lithographie von Albrecht von Haller filius ausgestattet, unter die durch Kombination der Namenszug Kasthofers gesetzt worden ist. Unter der Vorlage in Bern aber stehen als Schriftzüge die genannten Verse. Sie sind gleichzeitig mit dem Porträt lithographiert worden und zeigen Hallers, nicht Kasthofers Handschrift. So droht man Haller um das einzige Bildnis zu bringen, das ihn vergegenwärtigt. Für Mithilfe in der Bemühung, dies richtigzustellen, danke ich Herrn Dr. Hans Haeberli von der Burgerbibliothek Bern.

Heinz Balmer 